\title{
Sergei Duzhin (June 17, 1956-February 1, 2015)
}

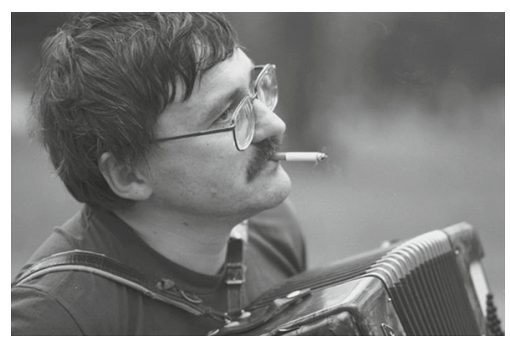

Photo by Nicolai Kitanine. Tokyo, February 23, 2002

Sergei Duzhin, a permanent participant of Arnold's seminar at the MSU for more than 20 years and a member of Arnold's school, unexpectedly passed away on February 1, 2015 because of an acute heart failure. This is our tribute to his memory.

Sergei Duzhin was born on June 17, 1956 in David-Gorodok, Belarus. In 1957, his family moved to Mogilev, Belarus, where he lived until graduating from the high school in 1972. He completed school studies in 9 years instead of the regular 10, due to the talent he demonstrated in his studies. Neither of his parents had anything to do with mathematics.

In 1972, he was accepted to Moscow State University (mekhmat), from which he graduated with an MS degree in Mathematics in 1978; then he continued his studies in the Ph.D. program at the same university under the guidance of Prof. Alexander Vinogradov. He was awarded Ph.D. degree in 1983 with the thesis On some versions of the $C$-spectral sequence dealing with the study of algebro-topological invariants of partial differential equations and foliations. Sergei returned to Mogilev in 1982 to a postdoc position of Assistant professor at the Mogilev Pedagogical Institute.

During the Mogilev years he, jointly with B. Chebotarevsky, wrote the book From Ornaments to Differential equations published by the Belarus publishing house "Vyshejshaya Shkola" in 1988 and translated into English under the title Transformation groups for Beginners, AMS Student Mathematical Library, v.25, 2004. 
In 1985, Sergei moved to Pereslavl-Zalessky (Yaroslavl region, Russia) to join Vinogradov's Laboratory of High-Dimensional Problems at the newly born Program Systems Institute of the Russian Academy of Sciences. As a member of Vinogradov's team, Sergei Duzhin participated in writing the book Symmetries and conservation laws for differential equations of mathematical physics, published in Russian in 1997 and in English in 1999, under the collective nom de plume of Jet Nestruev. Since 1986, he was a Senior Researcher and since 1991, after Vinogradov left the institute, headed the laboratory.

In Pereslavl-Zalessky, Sergei also became a member of a small, but mathematically active group of Arnold's former students. At about the same time, he started to attend Arnold's seminar at Moscow State University and soon became a very active participant there. Soon after the group became interested in low-dimensional topology, first of all in Vassiliev's theory of knot invariants, newly born at Arnold's seminar, praised and popularized by Vladimir Arnold. This theory became Sergei's passion for the rest of his life, and after becoming head of Vinogradov's former laboratory, he reoriented it and renamed Laboratory of Low-Dimensional Problems.

Vassiliev knot invariants of order $\leqslant n$ form a finite dimensional vector space $\mathcal{V}_{n}$ included into an increasing filtration

$$
\mathcal{V}_{0} \subseteq \mathcal{V}_{1} \subseteq \mathcal{V}_{2} \subseteq \cdots \subseteq \mathcal{V}_{n} \subseteq \mathcal{V}_{n+1} \subseteq \cdots \subseteq \mathcal{V}:=\bigcup_{n=0}^{\infty} \mathcal{V}_{n}
$$

Sergei's first result in this area, obtained jointly with [7], was an upper bound for the dimensions of the quotient spaces, $\operatorname{dim} \mathcal{V}_{n} / \mathcal{V}_{n-1} \leqslant(n-1)$ !. This paper sparked interest to the problem and gave rise to a series of studies that improved Sergei's initial result.

In the process of work on Vassiliev invariants, Sergei made enormous computations by hand, calculating all Vassiliev invariants up to degree 6 . He made two important observations. The first one is that the value of the symbol of an invariant, as a function on chord diagrams, depends on the intersection graph of a chord diagram rather than on the diagram itself. He formulated the intersection graph conjecture, which states that this is always true. Later it turned out that the conjecture fails in degree 11 and higher (T. Le), but the question about what part of information about a chord diagram is encoded in its intersection graph remains the subject of ongoing research.

His second observation was that the chromatic polynomial of the intersection graph satisfies the four-term relation. As a consequence, the chromatic polynomial must be a symbol of an appropriate Vassiliev invariant.

The development of these observations led to three papers (joint with S. Chmutov and S. Lando), usually referred to as [CDL], published in one issue of the Advances in Soviet Mathematics in 1994 [8-10]. (Initially, the papers were coauthored by the three persons in different combinations, but when the work had been completed, it became clear that it is impossible to separate contributions of each one to each of the papers). The first of them served as an introduction to the theory. In the second one, the intersection graph conjecture was proved for trees. (In the year 2000, B. Mellor proved the conjecture for chord diagrams whose intersection graph has a single cycle). In the 
last one, the notion of chromatic polynomial was extended to graphs with weighted vertices. In this case, it is a polynomial in several variables. It specializes to the classical chromatic polynomial in $x$ if the weights of all the vertices are set to be 1 and all the variables are set to $\pm x$. In 1999, S. Noble and D. Welsh noted that this polynomial is equivalent to the symmetric chromatic polynomial introduced by R. Stanley in 1995. There is a still an open conjecture of R. Stanley that this polynomial distinguishes trees. Thus the weighted chromatic polynomial introduced by Chmutov, Duzhin, and Lando in 1994 is directly related to this famous conjecture about trees. Also this paper contains a first lower bound for the number of independent Vassiliev invariants. In particular, it implies that there is at least one primitive Vassilev invariant in each degree.

In 1996, S. Duzhin obtained a quadratic lower bound for the dimensions of primitive spaces of Vassiliev invariants. Later, in 1999, jointly with S. Chmutov, he discovered a method for proving that this dimension grows faster than any polynomial. The method used the Lie algebra $\mathfrak{g l}_{N}$ and all its representations [12]. The same method was used by O. Dasbach to improve the lower estimate to $e^{c \sqrt{n}}$ in 2000. Note however, that the precise leading term of the asymptotics of dimensions of spaces of primitive elements is still out of reach.

In the 1990s, Sergei Duzhin started to travel a lot. In particular, he spent several years in Japan working at the universities of Tokyo and Aizu. During this period he published a survey article [11] (joint with S. Chmutov) about Arnold's invariants of plane immersed curves, a paper about the algebra of 3-graphs [13] (joint with S. Chmutov and A. Kaishev), a survey article (joint with S. Chmutov) about the Kontsevich integral [16]. In all his trips he communicated a lot with various peoplean occasional neighbor in an airplane seat, as well as some colleague working on problems in very far off domains. Sergei's remarkable talent for languages simplified communications a lot. In his numerous trips, he soon added to English, French, and Ancient Greek that he knew from the university years, also Italian, Japanese, Czech, and several others. His habit was to carry an accordion with him and to sing Soviet songs in any circumstances; this also attracted people, especially young ones. Sergei's love of vocal music and his talent as a communicator resulted in him being, in the last few years, the only mathematician to regularly communicate with Grigory Perelman.

In 2001, Sergei Duzhin moved to St. Petersburg, joining the Laboratory of representation theory and dynamical systems (A. M. Vershik's lab) at the St. Petersburg Division of the Steklov Mathematical Institute. There he found new weight systems for Vassiliev invariants related to the Klein equation and Plücker relation. He published two papers on this topic [17, 18] in 2002 and 2003. Jointly with J. Mostovoy he introduced a notion of finite type invariants for co-knots, that is, smooth functions $\mathbb{R}^{n} \rightarrow \mathbb{R}$ with a prescribed behavior at infinity. They published a paper on this subject [20] in 2006.

In 2007, jointly with his student M. Karev, he found a degree 7 Vassiliev invariant of long (string) links with two numbered components which is sensible to orientation reversal [21]. The proof is based on the study of a weight system with values in the 
tensor square of the universal enveloping algebra for the Lie algebra $\mathfrak{g l}_{N}$. In 2012 he published a paper [22] about the Conway polynomial and the Magnus expansion.

The same year the book (joint with S. Chmutov and J. Mostovoy) Introduction to Vassiliev Knot invariants [34] was published by Cambridge University Press. The book is a real mathematical and pedagogical masterpiece, describing in accessible terms the current state and open problems in this extremely sophisticated and beautiful theory, part of which is the result of Duzhin's research.

Also about the same time, Sergei, jointly with M. Shkolnikov (another student of his) proved a conjecture by J. Przytycki, which had stayed open for 24 years, notwithstanding the effort of several excellent mathematicians, including its author and J. H. Conway. Two papers $[23,26]$ were devoted to the proof.

The last published paper [25] of Sergei was joint work with D. Pasechnik and dealt with sandpile groups.

From the very beginning of Duzhin's work in St. Petersburg, he organized a Moscow-St. Petersburg seminar on Low-Dimensional Topology. This was the moment in the middle of a long 20-year period when the intensity of communications between the math communities of the two Russian capitals was at a very low level. Duzhin's seminar, which continued to work until his death, served as a pipe of fresh air between the capitals, allowing Moscow mathematicians to communicate their results to their Saint Petersburg colleagues and to become aware of current progress in the second capital. The crucial thing there was Sergei's successful efforts in obtaining financial support for travel expenses and hotel expenses for Moscow colleagues; when hotel expenses could not be covered, many mathematicians stayed in the guest room of his hospitable and comfortable apartment in the historic center of Saint Petersburg.

During all his mathematical life, Sergei Duzhin spent a lot of effort aimed at teaching school as well as university students. Already in the beginning of 1980s, when he was a graduate student of Alexander Vinogradov, he took an active part in teaching, together with his advisor, at the so-called "Jewish people's university" in Moscow. The aim of this informal institution was to teach mathematics to those interested students who were not accepted to the MSU mekhmat, due to their Jewish origin or for other non-academic reasons. Classes were given either at private apartments, or not very officially at various university auditoria. For more than three years, before the unavoidable crash of the university, many distinguished Moscow professors taught dozens of talented students, quite a few of whom play now an active role in developing math all over the world. In the early years of the Pereslavl-Zalessky Program Systems Institute, when even the simplest working and living facilities were not available, Sergei conducted math circles at home and taught school children at the Institute Summer Computer camp. (This is the very same Summer camp where the book Graphs on Surfaces and Their Applications by S. Lando and A. Zvonkin was initiated). 
In the 7-year period from 1998 to 2004, Duzhin taught six one-semester courses at the Independent University of Moscow and did the exercise classes in the calculus course for IUM freshmen in the academic year 1997-1998. Four of the courses were about various original and interesting aspects of combinatorics ${ }^{1}$, the other two were related to Vassiliev invariants and later formed the basis of several parts of the famous "CD-book" [34]. To do this, Sergei had to travel each week to Moscow from Pereyaslavl (later from Saint Petersburg), combining his teaching with participation in Arnold's seminar. He was the only non-Moscovite to teach regularly at the IUM for many years.

In St. Petersburg, Duzhin soon began to play a crucial role in the mathematical part of the Physics and Mathematics Club (Fizmatclub), where students of many city universities used to gather on weekends to study those parts of modern science they were unable to grasp at their home universities. Sergei's contribution consists in a number of original well-developed lecture courses. In his late years, this type of activity widened to popular lectures for the general public, like the one devoted to the Poincaré conjecture and Perelman's proof of it.

One of his favorite phrases was from an obituary of L. Euler "... he ceased to calculate and to live". It can be applied to Sergei himself. Up to the very last day of his life, Sergei performed calculations, and his calculations were interrupted by his death only.

He is survived by his wife Olga Muravtsova and two sons Fedor and Vassili, his sister Elena and brother Alexander.

S. Chmutov, M. Karev, A. Khovanski, J. Krasilshchik, S. Lando, N. Mnev, G. Panina, Yu. Romanovski, I. Scherbak, A. Sossinski, A. Varchenko, N. Vasilyev, V. Vassiliev, A. Vershik

\section{Sergei Duzhin's publications}

\section{Papers published in referred journals}

1. Duzhin, S.V.: A $C$-spectral sequence on the manifold $J^{1}(M)$. Russian Math. Surveys 38(1), 179-181 (1983)

2. Duzhin, S.V.: A spectral sequence associated with a foliation, and Gelfand-Fuks cohomology of certain Lie algebras of vector fields. Russian Math. Surveys 39(1), 147-148 (1984)

3. Duzhin, S.V., Tsujishita, T.: Conservation laws of the BBM equation. J. Phys. A Math. Gen. 17, 3267-3276 (1984)

4. Duzhin, S.V., Lychagin, V.V.: Symmetries of distributions and quadrature of ordinary differential equations. Acta Appl. Math. 24, 29-57 (1991)

5. Duzhin, S.V., Chmutov, S.V.: Gaydar's formula for the greatest common divisor of several multivariate polynomials. Russian Math. Surveys 48(2), 171-172 (1993)

\footnotetext{
1 Lecture notes and other materials about these courses are available on Sergei's websites http://www.pdmi. ras.ru/ duzhin/ and http://www.botik.ru/ duzhin/NMU/.
} 
6. Carrà-Ferro, G., Duzhin, S.V.: Differential-algebraic and differential-geometric approach to the study of involutive symbols. In: Ibragimov, N.H., et al. (eds.) Modern Group Analysis: Advanced Analytical and Computational Methods in Mathematical Physics, pp. 93-99. Kluwer, Dordrecht (1993)

7. Chmutov, S.V., Duzhin, S.V.: An upper bound for the number of Vassiliev knot invariants. J. Knot Theory Ramif. 3, 141-151 (1994)

8. Chmutov, S.V., Duzhin, S.V., Lando, S.K.: Vassiliev knot invariants I. Introduction. In: Arnold, V.I. (ed.) Adv. in Soviet Math., vol. 21. Singularities and Curves, pp. 117-126 (1994a)

9. Chmutov, S.V., Duzhin, S.V., Lando, S.K.: Vassiliev knot invariants II. Intersection graph conjecture for trees. In: Arnold, V.I. (ed.) Adv. in Soviet Math., vol. 21. Singularities and Curves, pp. 127-134 (1994b)

10. Chmutov, S.V., Duzhin, S.V., Lando, S.K.: Vassiliev knot invariants III. Forest algebra and weighted graphs. In: Arnold, V.I. (ed.) Adv. in Soviet Math., vol. 21. Singularities and Curves, pp. 135-145 (1994c)

11. Chmutov, S., Duzhin, S.: Explicit formulas for Arnold's generic curve invariants. In: Geometry and Singularity Theory, Arnold-Gelfand Mathematical Seminars, pp. 123-138. Birkhauser (1997)

12. Chmutov, S.V., Duzhin, S.V.: A lower bound for the number of Vassiliev knot invariants. Topology Appl. 92, 201-223 (1999)

13. Chmutov, S.V., Duzhin, S.V., Kaishev, A.I.: The algebra of 3-graphs. Trudy Matematicheskogo Instituta im. Steklova, vol. 221, pp. 168-196 (1998) [English translation: Proc. Steklov Inst. Math. 221(2), 157186 (1998)]

14. Duzhin, S.V., Kaishev, A.I.: T-system implementation of the program of computation of sl- and so-polynomials for 3-graphs. In: Programmnye Sistemy (transactions of Program Systems Institute, Pereslavl-Zalessky), Moscow, pp. 214-223 (1999, in Russian)

15. Duzhin, S.V.: Infinitesimal classification of systems of two first order partial differential equations. Zapiski nauch seminarov POMI 279 (2001) 61-69 (in Russian). English translation. J. Math. Sci. 119(1), 30-34 (2004)

16. Chmutov, S.V., Duzhin, S.V.: The Kontsevich integral. Acta Appl. Math. 66(2), 155-190 (2001)

17. Duzhin, S.: On the Kleinian weight systems. Low-dimensional topology of tomorrow. Suurikaisekikenkyuusho Kookyuuroku 1272, 84-90 (2002)

18. Duzhin, S.: Decomposable skew-symmetric functions. Moscow Math. J. 3(3), 881-888 (2003)

19. Chmutov, S., Duzhin, S.: The Kontsevich integral. In: Francoise, J.-P., Naber, G.L., Tsou, S.T. (eds.) Encyclopedia of Mathematical Physics, pp. 231-239. Elsevier, Oxford, ISBN 978-0-1251-2666-3 (2006)

20. Duzhin, S., Mostovoy, J.: A toy theory of Vassiliev invariants. Moscow Math. J. 6(1), 85-93 (2006)

21. Duzhin, S.V., Karev, M.V.: Detecting the orientation of string links by finite type invariants. Funktsional'nyi Analiz i Ego Prilozheniya 41(3), 48-59 (2007, in Russian) [English translation: Funct. Anal. Appl. 41(3), 208-216 (2007)]

22. Duzhin, S.: Conway polynomial and Magnus expansion. St. Petersburg Math. J. 23(3), 541-550 (2012)

23. Duzhin, S.: Proof of Przytycki's conjecture on matched diagrams. Doklady Math. 84(3), 787-788 (2011)

24. Duzhin, S.V.: Algorithms for the computations of the Conway polynomial from a bipartite graph. Inf. Control Syst. 4, 89-91 (2011)

25. Duzhin, S.V., Pasechnik, D.V.: Groups acting on necklaces and sandpile groups. Zapiski nauch seminarov POMI 421 (2014) 81-93 (in English). J. Math. Sci. 200(6), 690-697 (2014)

26. Duzhin, S., Shkolnikov, M.: Bipartite knots. Fundam. Math. 225, 95-102 (2014)

\section{Books}

27. Duzhin, S.V., Chebotarevsky, B.D.: From ornaments to differential equations (an introduction to the theory of transformation groups). Minsk, Vysheishaya Shkola (1988, in Russian)

28. Duzhin, S.V., Chebotarevsky, B.D.: Henkangun nyuumon. Springer, Tokyo (2000) (second printing (2002))

29. Duzhin, S.V., Chebotarevsky, B.D.: Transformation groups for beginners, AMS, Student Mathematical Library, vol. 25 (2004) 
30. Bocharov, A., Verbovetsky, A., Duzhin, S., et al.: In: Vinogradov, A., Krasilshchik, I. (eds.) Symmetries and Conservation Laws for Differential Equations of Mathematical Physics. Factorial Publishers, Moscow, ISBN 5-88688-019-4 (1997, in Russian)

31. Bocharov, A., Verbovetsky, A., Duzhin, S., et al.: In: Vinogradov, A., Krasilshchik, I.: AMS MMONO/182, ISBN 0-8218-0958-X (1999)

32. Jet Nestruev (group of authors consisting of Astashov, A., Bocharov, A., Duzhin, S., Sosinsky, A., Vinogradov, A., Vinogradov, M.): Smooth manifolds and observables. MCCME Publishers, Moscow (2000, in Russian)

33. Nestruev, Jet: Graduate Texts in Mathematics, vol. 220. Springer, Berlin (2002)

34. Chmutov, S., Duzhin, S., Mostovoy, J.: Introduction to Vassiliev Knot Invariants. Cambridge University Press, Cambridge, ISBN 978-1-107-02083-2 (2012)

\section{Popularization}

35. Duzhin, S.V., Rubtsov, V.N.: Geometry of the 4-dimensional cube. Kvant 6, 3-7 (1986). (in Russian)

36. Duzhin, S.: Seminar diary column in Suugaku seminar (Tokyo), a set of 12 essays, April 1996-March 1997 (in Japanese)

37. Duzhin, S.V., Chmutov, S.V.: Knots and their invariants. Matematicheskoe Prosveschenie 3, 59-93 (1999, in Russian)

38. Chmutov, S., Duzhin, S.: Kontsevich's integral. In: Encyclopaedia of Mathematics, Supplement II. Kluwer, Dordrecht (1999)

39. Duzhin, S.V.: Vassiliev-Goussarov invariants. In: Vershik, A.M. (eds.) Mathematics of XX century, view from St. Petersburg. MCCME Publishers, pp. 87-116 (2010, in Russian)

40. Duzhin, S.V.: What did Grigori Perelman prove? Troitsky Variant 10(104), 6-7 (2012, in Russian). http://trv-science.ru/2012/05/22/chto-zhe-dokazal-grigorijj-perelman/

41. Duzhin, S.V.: Grisha Perelman, apple, and donut. Lecture at Polit.ru (2012, in Russian). Video available at http://www.youtube.com/watch?v=4a7UeLRtxXY

42. Duzhin, S.V.: Enumeration of trees. Matematicheskoe Prosveschenie 16, 14-25 (2012). (in Russian)

43. Chmutov, S., Duzhin, S.: Kontsevich Integral. From MathWorld—A Wolfram Web Resource, created by Eric W. Weisstein. http://mathworld.wolfram.com/KontsevichIntegral.html

44. Duzhin, S.: Vassiliev Invariant. From MathWorld-A Wolfram Web Resource, created by Eric W. Weisstein. http://mathworld.wolfram.com/VassilievInvariant.html 